\title{
Non-specific Arm Pain
}

\author{
Vukasin Mihajlovic \\ Faculty of Applied Physiotherapy Igalo, University of Montenegro, Herceg Novi 85340, Montenegro
}

\begin{abstract}
Non-specific arm pain is a special clinical condition that can occur in work-related activities that involve maintaining a static position for prolonged periods or repetitive and frequent movements of the hand or entire arm. Such activities include typing on a keyboard, maneuvering a computer mouse, playing musical instruments (such as piano and guitar) and many forms of manual labor. The pain is dull and diffuse; It is localized in the forearm or in the hand but quickly can expand to the entire extremity. Non-specific arm pain is the most frequent type of work-related pain after lower-back pain. It thus has important socio-economic significance as a major cause of absence from work. The designation of "non-specific" originates from the fact that it has no obvious signs of tissue damage, unlike the "specific" pain accompanying carpal tunnel syndrome, tenosinovitis de Quervain, or lateral epicondylitis. Suggested causes of the pain include microtrauma of soft tissue followed by an inflammatory reaction, ischemia, fatigue, hyper-sensitization of nociceptors, focal dystonia of the hand and/or psychological stress. Treatment consists of application of NSAIDs, physical modalities, stretching and aerobic exercises. Prevention focuses on ergonomic modification during manual labor or work on a computer.
\end{abstract}

Key words: Absence of obvious signs of tissue damage, maneuvering a computer mouse, non-specific arm pain, typing on a keyboard, work-related upper limb disorders.

\section{Introduction}

The purpose of this article is to describe the clinical and socio-economic significance of non-specific arm pain, its difference from specific arm pain, and particularly the treatment and prevention of this common condition.

Non-specific arm pain is a special clinical condition that can occur in activities that involve repetitive movements, extreme positions, or maintaining a static position for prolonged periods. Common activities responsible for this condition include typing on a keyboard, maneuvering a computer mouse, playing musical instruments (such as piano and guitar), and many forms of manual labor.

These activities can lead to carpal tunnel syndrome, de Quervain tenosinovitis, lateral epicondylitis or tendonitis in the shoulders. These conditions are accompanied by pain classified as specific.

Patients with non-specific pain tissue damage have

Corresponding author: Vukasin Mihajlovic, M.D., Ph.D., professor of physiotherapy, research fields: physical therapeutic modalities in treatment of pain. no obvious signs of tissue damage, so the diagnosis of non-specific pain is considered diagnosis per exclusionem [1].

Non-specific arm pain is referred to as "repetitive strain injury" because it is assumed that the main cause of the pain is repetitive strain of soft tissue. This designation is debatable, although widely used because of the etiology and pathology of the condition and should be used as a synonym for specific impairments, such as carpal tunnel syndrome, tenosynovitis de Quervain, etc. [2]. Non-specific arm pain is the most frequent type of work-related pain after lower back pain [3]. An epidemic of this sort of pain was documented in Australia at the beginning of the 1980s. Interestingly, this event coincided with the widespread replacement of traditional typewriters with computer keyboard [4].

\section{Risk Factors}

Risk factors for developing non-specific arm pain include a poor ergonomic environment, a mechanically monotonous work routine, time-consuming work 
without a break, work involving continuously vibrating equipment, a lack of autonomy, and a high level of psychological stress in the work-place [5]. Women are more sensitive to these injuries.

\section{Mechanism of Pain}

The pain characteristic of this condition originates from repetitive microtrauma of muscles, tendons, nerves, and loose connective tissue from the initial reaction to the acute inflammation, subsequent degeneration, and fibrosis of the tissue; reorganization of the tissue results in the non-specific pain [6]. Inflammation indicates an increased systemic levels of inflammatory mediators in the serum (interleukin-1 $\beta$, factor- $\alpha$ tumor necrosis factor, interleukin- 6 , C-reactive protein) [7].

Ischemia is another suspected cause of pain. Isometric contraction reduces the flow of blood and, consequently, oxygen to the forearm muscles of patients with this condition [8]. There also is evidence that some patients have increased intrafascial pressure in the forearm extensor compartment. This reduces blood flow and causes muscle spasms during continuous repetitive activity [9]. Muscle biopsy samples showed hypertrophic type I muscle fibers and mitochondrial dysfunction. This is considered to indicate muscle ischemia [10]. Muscle fatigue also can be sources of pain [11].

Pain can be of neurogenic origin. Repeated stimulation of nociceptors can cause hyper-sensitization and expand the receptive field of nociceptors. This, in turn, can increase the excitability to the second level neurons. Additionally, there is a possibility of reorganization in the somatosensory cortex level with the emergence of focal dystonia of the hand [5]. Some authors believe that this non-specific pain is related to psychological stress rather than the mechanics of physical activity $[12,13]$.

\section{Clinical Presentation}

Diffuse, dull pain in the forearm or hand is the predominant symptom. In the beginning of activity it is intermittent then eases with rest. Its intensity increases with continuing activity and can be triggered by minimal activity in the work-place or even by the activities of daily living as dressing, etc. The pain initially is localized in the forearm or the hand but quickly can expand to the entire extremity and, eventually, to the other arm. It increases gradually in such a way that, by the end of the day, it reaches its maximum intensity. The pain and other symptoms do not follow a dermatological or peripheral nerve distribution.

Other symptoms are paresthesia, stiffness, nausea, cramps, allodynia and reduced of the fine movements of the hand. Furthermore, patients often complain of nocturnal pain, insomnia, anxiety and depression [14].

\section{Treatment}

The initial treatment consists of stopping activities that cause the pain and immobilizing the affected area. This is followed by application of ice, TENS (transcutaneous electrical nerve stimulation), interference current, ultrasound, iontophoresis of local anesthetics and analgesics, and oral application of non-steroidal anti-inflammatory medications. If there is a sleep disorder and/or depression, tricyclic antidepressants are administered in small doses [15].

The post-acute phase features stretching, strengthening, progressive resistance exercises and aerobics. Prescription of therapeutic exercise naturally must take into account any biomechanical abnormalities that might afflict a patient.

\section{Prevention}

Prevention focuses on ergonomic modifications. For example, when working on a computer, the chair should be adjusted so that the forearms rest horizontally on the table with the hands in a neutral position. The shoulders should be relaxed. The keyboard should be directly in front of the user to prevent any ulnar deviations. The mouse should be 
near the middle [15]. The head should not be tilted up in relation to the monitor; the monitor should be directed downwards at an angle of $18^{\circ}$ and $24^{\circ}$. The lumbar region should be supported by the back of the chair. The knees should be bent at a $90^{\circ}$ angle with the feet on the floor or placed on a foot-rest. Several short breaks over the course of a work session prove better than one long break.

Short breaks are of importance for the prevention of non-specific arm pain as indicated by the fact that it does not appear when typing on traditional typewriters because working on traditional typewriters includes intermittent breaks: That is, after typing each line the typist must manually advance the paper and regularly insert a new sheet of paper and usually carry the finished document somewhere from his desk. In contrast, typing on a computer is performed more continuously: There is no need for carriage returns, inserting new sheets, manual copying, and the completed document is electronically dispatched to its destination [16].

Particularly risky tasks should be scheduled in a way that rotates workers' participation, the weight of heavy objects should be reduced, and the distance over which objects are moved should be shortened. Temperature and lighting should be optimal and the work area should be well-ventilated. Very intensive activities should be short and feature regular breaks.

\section{Conclusions}

Non-specific arm pain has become a very commonly reported syndrome. It is associated mainly with computer work and has emerged as a significant economic problem owing to its role in work-place absences and the high cost of treatment. Because it is not accompanied by obvious tissue damage, diagnosis relies on exclusion of other arm disorders. Early diagnosis and treatment are key to recovery. Treatment consists of discontinuing work-related activities, medications and physical therapy. Prevention is based on education in ergonomic practices.

\section{References}

[1] Gold, J. E., Piligian, G., Glutting, J. J., Hanlon, A., Frings-Dresen, M. H., and Sluiter, J. K. 2010. "Cluster Analysis of Symptoms among Patients with Upper Extremity Musculoskeletal Disorders." J. Occup. Rehabil. 20 (4): 526-36.

[2] Dilley, A., and Greening, J. 2014. "Non-specific Arm Pain." In Wall and Melzack's Text-Book of Pain, edited by McMahon, S. B., Koltzenburg, M., Tracy, I., and Turk, D. C. Elsevier Saunders.

[3] Silverstein, B., Welp, E., Nelson, N., and Kalat, J. 1998. "Claims Incidence of Work-Related Disorders of the Upper Extremities.” Am. J. Public Health 88 (12): 1827-33.

[4] Ferguson, D. A. 1987. "RSI Putting the Epidemic to Rest." Med. J. Aust. 147 (5): 213-4.

[5] Barr, A., Barbe, M., and Clark, B. 2004. "Work-Related Musculoskeletal Disorders of the Hand and Wrist: Epidemiology, Pathophysiology, and Sensorimotor Changes." J. Orthop. Sports Phys. Ther. 34 (10): 610-27.

[6] Barr, A., and Barbe, M. 2002. "Pathophysiological Tissue Change Associated with Repetitive Movement: A Review of Evidence." Phys. Ther. 82 (2): 173-87.

[7] Carp, S., J., Barbe, M. F., Winter, K. A,. Amin, M., and Barr, A. E. 2007. "Inflammatory Biomarkers Increase with Severity of Upper-Extremity Overuse Disorders." Clin. Sci. (Lond) 112 (5): 305-14.

[8] Brunnekreef, J., Oosterhof, J., Thijssen, D., Colier, W. N., and Van Uden, C.J. 2006. "Forearm Blood Flow and Oxygen Consumptition in Patients with Bilateral Repetitive Strain Injury Measured by Near-Infrared Spectroscopy." Clin. Physiol. Funct Imaging 26 (3): 178-84.

[9] Pritchard, M. H., Williams, R. L., and Heath, J. P. 2005. "Chronic Compartment Syndrome, an Important Cause of Work-Related Upper Limb Disorder." Rheumatology 44 (9): 1442-6.

[10] Dennett, X., and Fry, H. J. 1988. "Overuse Syndrome: A MuscleBiopsy Study.” Lancet 1 (8591): 905-8.

[11] Helliwell, P., and Taylor, W. 2004. "Repetitive Strain Injury." Postgrad Med. J. 80 (946): 438-43.

[12] Walker-Bone, K., and Cooper, C. 2005. "Hard Work Never Hurt Anyone or Did It? A Review of Occupational Associations with Soft Tissue Musculoskeletal Disorders of the Neck and Upper Limb." Ann. Rheum Dis. 64 (10): 1391-6.

[13] Walker-Bone, K., Reading, I., Coggon, D., Cooper, C., and Palmer, K.T. 2004. "The Anatomical Pattern and Determinants of Pain in the Neck and Upper Limbs: An 
Epidemiologic Study.” Pain 109 (1-2): 45-51.

[14] Ring, D., Radzielski, J., Fabian, L., Zurakowski, D., Malhotra, L. R., and Jupiter, J. B. 2006. "Self-reported Upper Extremity Health Status Correlates with Depression.” J. Bone Joint Surg. Am. 88 (9): 1983-8.

[15] McInnis, K. 2008. "Repetitive Strain Injury." In
Essentials of Physical Medicine and Rehabilitation, edited by Frontera, W. R., Silver, K., and Rizzo Jr, T. D. Elsevier Saunders.

[16] Lynn, B. 2006. "Repetitive Strain Injury." In Wall and Melzack's Textbook of Pain, edited by McMahon, S. B., and Koltzenburg, M. Elsevier Churchill Living-Stone. 